\title{
Age and individual differences in visuospatial processing speed: Testing the magnification hypothesis
}

\author{
YINGYE ZHENG, JOEL MYERSON, and SANDRA HALE \\ Washington University, St. Louis, Missouri
}

\begin{abstract}
Forty young adults and $\mathbf{4 0}$ older adults performed seven visuospatial information processing tasks. Factor analyses of the response times (RTs) yielded a single principal component with a similar composition in both age samples. For both samples, regressing the mean RTs of fast and slow subgroups for the seven tasks ( 18 conditions) on the corresponding mean RTs for their age group accounted for $99 \%$ of the variance. Taken together, these findings suggest that individual differences in processing time were largely task independent. The magnification hypothesis, a simple mathematical model of the interaction between age and ability, is presented. This model correctly predicts the finding that in both the young and the older adult groups, individual differences increased systematically with task difficulty. The magnification hypothesis also explains the regression parameters describing individual differences among young adults and predicts correctly that equivalent parameters describe individual differences among older adults. According to the magnification hypothesis, the RTs of slower individuals are more affected by aging than those of faster individuals, and slower individuals may be more at risk with respect to other biological insults (e.g., changes in health status) as well.
\end{abstract}

Processing speed measures have proven useful in studying how age (see, e.g., Cerella \& Hale, 1994; Kail \& Salthouse, 1994) and ability (e.g., Hale \& Jansen, 1994; Miller \& Vernon, 1992) affect cognitive performance. In young adults, factor analysis of response time (RT) data typically reveals a single, general factor that accounts for more than half of the variance (see, e.g., Miller \& Vernon, 1992; Vernon \& Jensen, 1984). Such factor analytic findings, however, reveal little about the absolute magnitude of individual differences on different tasks. The question of the size of individual differences can be addressed using regression analysis, however, as exemplified by the approach of Hale and Jansen (1994). These researchers tested young adults on seven different information processing tasks and then regressed the RTs of subgroups of fast and slow individuals on the group mean RTs for the corresponding tasks and conditions. The results revealed that individual differences in RTs increase as the amount of processing increases regardless of the type of processing that is required (Hale \& Jansen, 1994).

Despite the extensive research on age-related cognitive slowing (Cerella \& Hale, 1994), it is not generally realized how little examination of differences between the mean

\footnotetext{
A preliminary report of this study was presented at the annual meeting of the Psychonomic Society, Chicago, November 1996. Y.Z.'s efforts were supported in part by a Student Research Award from Division 20 (Adult Development and Aging) of the American Psychological Association. Additional support was provided by the National Institute on Aging (Grant ROI AG12996) to S.H. Correspondence should be addressed to J. Myerson, Department of Psychology, Campus Box 1125, Washington University, St. Louis, MO 63130 (e-mail: jmyerson@ wastl.artsci.edu)
}

RTs of different age groups reveals about individuals. For example, the typical complexity effect, in which the age difference between mean RTs increases with task difficulty (Cerella, Poon, \& Williams, 1980), might occur because the size of the age deficit is larger on more difficult tasks or because the proportion of individuals showing an age deficit is larger on more difficult tasks. Other explanations are obviously possible, and explicit testing of such hypotheses regarding the effects of aging on individual performances is clearly needed (Maylor \& Rabbitt. 1994).

Although there has been relatively little direct investigation of how aging affects individual differences in cognition, several quite different lines of evidence are relevant to this issue. First, the ranking of individual young adults on speeded tasks is quite stable from task to task, but the size of individual differences in RTs increases with task difficulty (Hale \& Jansen, 1994). Second, psychometric data indicate that the ranking of healthy individuals in a cohort is highly stable over the adult years (Hertzog \& Schaie, 1986; Schaie, 1989). Third, speeded cognitive performance is almost always more diverse among older adults than among young adults (i.e., the standard deviation of the RTs of an older adult group is larger than the standard deviation for a young adult group performing the same task). This difference is primarily due to age-related slowing, however, as indicated by the fact that there is no age difference in diversity when speed differences are statistically controlled (Hale, Myerson, Smith, \& Poon, 1988).

These findings lead us to propose a magnification $h y$ pothesis. According to this hypothesis, general age-related 
slowing operates at the individual level, magnifying everyone's processing times and increasing the size of individual differences by the same task-independent factor. For present purposes, we define the processing time of the $i$ th individual as $\mathrm{RT}_{i}-b$, where $b$ represents the time required for response selection and execution. Following Hale and Jansen (1994), we hypothesize that individual differences between young adults (as indicated by the subscript $y$ ) may be represented by a task-independent processing time coefficient, $c_{i}$, that indicates the degree to which an individual's processing is slower $\left(c_{i}>1.0\right)$ or faster $\left(c_{i}<1.0\right)$ than average:

$$
\mathrm{RT}_{i, \mathrm{y}}-b=c_{i}\left(\mathrm{RT}_{\mathrm{avg}, \mathrm{y}}-b\right) .
$$

Rearranging reveals that an individual young adult's RTs on different tasks will be a simple linear function of the corresponding group mean RTs,

$$
\mathrm{RT}_{i, \mathrm{y}}=c_{i}\left(\mathrm{RT}_{\mathrm{avg}, \mathrm{y}}\right)+b\left(1-c_{i}\right)
$$

with slopes greater than 1.0 and negative intercepts for slow individuals and slopes less than 1.0 and positive intercepts for fast individuals (Hale \& Jansen, 1994).

The magnification hypothesis further assumes that for a group of older adults, the processing time of each individual on each task (i.e., the difference between the RT and the time required for response selection and execution) is magnified by the same age-related cognitive slowing factor, $s$. Expressed mathematically,

$$
\mathrm{RT}_{i, \mathrm{0}}-b=s\left(\mathrm{RT}_{i, \mathrm{y}}-b\right),
$$

where the age group is indexed by the subscript (o indicates older). It follows immediately that

$$
\mathrm{RT}_{\text {avg }, \mathrm{o}}-b=s\left(\mathrm{RT}_{\text {avg,y }}-b\right) \text {. }
$$

Dividing both equations (i.e., Equations 3 and 4 ) by $s$ and substituting into Equation 1 yields

$$
\left(\mathrm{RT}_{i, \mathrm{o}}-b\right) / s=c_{i}\left(\mathrm{RT}_{\text {avg }, o}-b\right) / s .
$$

Rearrangement and simplification of Equation 5 yields

$$
\mathrm{RT}_{i, \mathrm{o}}=c_{i}\left(\mathrm{RT}_{\mathrm{avg}, \mathrm{o}}\right)+b\left(1-c_{i}\right)
$$

It can be seen that Equation 6 is equivalent to Equation 2, revealing that despite age-related slowing, the predicted relation between individual and average RTs is the same for both young and older adults.

Thus, the magnification hypothesis (in essence, Equations 1 and 3 ) leads to strong predictions concerning the RTs of matched young and older individuals or subgroups (i.e., individuals of equivalent ability for their age). That is, the slope and intercept for the regression of fast young RTs on average young RTs from the same tasks and conditions should not differ significantly from the slope and intercept for the regression of fast old RTs on average old RTs, and a similar equivalence should hold for the regression parameters for slow young and old RTs.

The magnification hypothesis also predicts the results of a pioneering study by Maylor and Rabbitt (1994). These researchers examined the interaction of age and individual differences on two speeded paper-and-pencil tasks, visual search and letter coding (a task analogous to digit-symbol substitution). They found that for each of the tasks they studied, the RTs of one age group (75-year-olds), ranked from fastest to slowest, were a linear function of the ranked RTs of another, younger age group (60-year-olds). The slope of the regression was greater than 1.0 for both tasks, indicating that in each case the age difference in RTs increased systematically from the fastest to the slowest individuals.

The magnification hypothesis prediction of these findings is obtained by simply rearranging Equation 3 to obtain

$$
\mathrm{RT}_{\mathrm{o}, i}=s \mathrm{RT}_{\mathrm{y}, i}-b(s-1) .
$$

The preceding equation accounts for the linear relation between the RTs of older individuals and those of corresponding younger individuals (whose performance serves as proxy for the performance of the older individuals when they were younger). The slope of this relation is greater than 1.0 because it is equal to the age-related cognitive slowing factor, $s$.

Although the Maylor and Rabbit (1994) results follow from the assumptions of the magnification hypothesis, it is not possible to reverse this logic and derive these assumptions from the observed linear relations between the RTs of corresponding older and younger adults on specific tasks. This is because when RTs are analyzed separately for each task, as was the case in the Maylor and Rabbitt study, fast and slow individuals on one task need not be fast and slow on other tasks. As a consequence, the systematic relations observed on each task provide no clues as to how individual differences change from task to task.

In contrast, the magnification hypothesis makes strong assumptions regarding how individual processing times are affected first by task difficulty, as indexed by the group mean RT (Equation 1), and again by aging (Equation 3). These assumptions lead directly to predictions of linear relations between individual and average performance across multiple tasks and conditions and, most importantly, to the unique prediction that such relations are unaffected by aging. These predictions are the focus of the present study, which, to the best of our knowledge, is the first to investigate the effects of aging on the magnitude of individual differences in RTs across multiple tasks. Young and older adults were tested on seven different visuospatial tasks, and the relations between the RTs of individual participants or subgroups of participants of similar ability (e.g., fast or slow individuals) and the mean RTs for their age group were examined in order to evaluate the predictions of the magnification hypothesis.

\section{METHOD}

\section{Participants}

Forty young ( $M$ age $=21.4$ years, $S D=1.0$ ) and 40 older adults ( $M$ age $=71.2$ years, $S D=3.4$ ) participated in the study; there were 21 females and 19 males in each group. The young adults were re- 

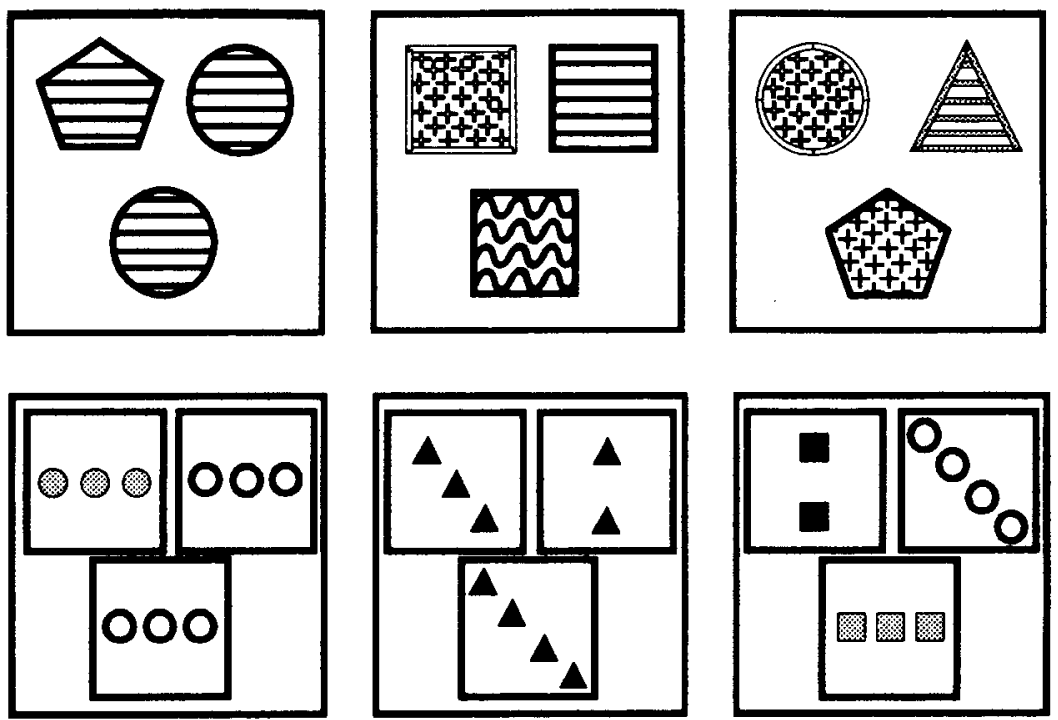

Figure 1. Sample stimuli for the two abstract matching tasks. The top panel depicts stimuli for the three conditions of Abstract Matching 1 and the bottom panel depicts stimuli for the three conditions of Abstract Matching 2. There were three conditions for each task, corresponding to the sample stimuli in the left, middle, and right positions of each row: Level 1, both irrelevant dimensions held constant; Level 2, one irrelevant dimension held constant and one varied; Level 3, both irrelevant dimensions varied.

cruited from the pool of undergraduate volunteers maintained by the Washington University Department of Psychology. Each received $\$ 5$ for participation. The older adults were recruited from the pool of volunteers maintained by the Adult Aging and Development Program in the Department of Psychology at Washington University. Each received $\$ 10$ for participation and travel expenses. The education level of the older adult group ( $M$ education $=$ 14.7 years, $S D=2.7)$ was comparable to that of the young adult group ( $M=15.1$ years, $S D=1.0$ ). All participants were righthanded, rated their health as good to excellent, and had normal or corrected-to-normal visual acuity based on their ability to read a standard eye chart at a distance of $20 \mathrm{ft}$.

\section{Apparatus}

Stimuli were presented on an NEC MultiSynch $2 \mathrm{~A}$ color monitor controlled by an IBM-compatible 286 computer running software written in Turbo Pascal by S.H. Presentation was synchronized with the video refresh cycle, and RTs were recorded with 1-msec accuracy using display and timing routines from the PCX Toolkit by Genus. Typical viewing distance was approximately $60 \mathrm{~cm}$. A response panel housed three buttons arranged in an inverted triangle. Participants used the upper left and right buttons to report decisions and the lower, center button to initiate trials.

\section{Tasks}

Participants were administered seven visuospatial information processing tasks that were presented in the following order: the three-object task, the one-arrow task, the Abstract Matching Task 1, the one-object task, the two-object task, the Abstract Matching Task 2, and the two-arrow task. The same order was used for all participants in order to avoid confounding order effects with potential individual strengths and weaknesses, and the specific order used was selected so as to avoid confounding the effects of fatigue and practice with those of task complexity. A brief description of each task follows. Stimulus dimensions are given in degrees of vi- sual angle, calculated on the basis of the typical viewing distance $\left(1.05 \mathrm{~cm}=1^{\circ}\right)$.

One-arrow task. The stimulus was an arrow $\left(1.0^{\circ} \times 2.4^{\circ}\right)$ pointing either left or right. Participants were instructed to press the button that corresponded to the direction of the arrow.

Two-arrow task. The stimulus was two arrows (each $3.0^{\circ} \times 0.5^{\circ}$ and separated by $0.7^{\circ}$ ) that pointed in either the same or opposite directions. To avoid the possibility that the stimuli could provide internally inconsistent information as to whether a right or left response was required, especially in the context of the previous one-arrow task, the arrows in the present task were of a different size and were oriented vertically. Participants were instructed to press the left button if the directions were the same and the right button if they differed.

One-object task. The stimulus was either a blue square $\left(1.9^{\circ}\right.$ sides) or pink circle ( $1.9^{\circ}$ diameter). Participants were instructed to press the left button if the blue square appeared and the right button if the pink circle appeared.

Two-object task. The stimulus consisted of two objects (each $1.9^{\circ} \times 1.9^{\circ}$ ). Each object was either a square or a circle, and was green, yellow, blue, or red. Participants were instructed to press the left button if the shapes were the same and the right button if they were different.

Three-object task. The stimulus consisted of three objects (each $1.9^{\circ} \times 1.9^{\circ}-2.3^{\circ}$ ) outlined in green and unfilled. Each object had one of six shapes (circle, oval, square, rectangle, equilateral triangle, and right triangle). Participants were instructed to press the left button if two of the three objects were the same shape and to press the right button if all three were different shapes.

Abstract Matching Task 1. The stimulus consisted of three objects (each approximately $4.3^{\circ} \times 4.3^{\circ}$ ). Participants were instructed to indicate which of the upper two objects best matched the bottom object (Figure 1, top panel) by pressing the corresponding button. Each object varied on three dimensions: shape (circle, triangle, pentagon, or square), color (red, blue, green, or yellow), and texture (straight lines, wavy lines, crosses, or dashes). On any given trial, 
Table 1

Mean Response Times (RTs, in Milliseconds), Standard Deviations (SD), and Error Rates (\%)

\begin{tabular}{|c|c|c|c|c|c|c|}
\hline \multirow[b]{3}{*}{ Conditions } & \multicolumn{6}{|c|}{ Group } \\
\hline & \multicolumn{3}{|c|}{ Younger Adults } & \multicolumn{3}{|c|}{ Older Adults } \\
\hline & $\mathrm{RT}$ & $S D$ & $\%$ Error & RT & $S D$ & $\%$ Error \\
\hline Arrow direction & 380 & 77 & 0.2 & 541 & 136 & 0.0 \\
\hline One object & 453 & 86 & 1.8 & 645 & 166 & 0.8 \\
\hline \multicolumn{7}{|l|}{ Disjunctive choice } \\
\hline Same & 542 & 91 & 4.0 & 771 & 146 & 2.0 \\
\hline Different & 575 & 90 & 2.0 & 748 & 130 & 0.4 \\
\hline \multicolumn{7}{|l|}{ Two objects } \\
\hline Same color/same shape & 489 & 89 & 1.7 & 691 & 162 & 1.2 \\
\hline Same color/different shape & 519 & 92 & 2.1 & 728 & 141 & 1.4 \\
\hline Different color/same shape & 520 & 96 & 1.0 & 718 & 145 & 0.5 \\
\hline Different color/different shape & 552 & 107 & 2.0 & 762 & 174 & 0.6 \\
\hline \multicolumn{7}{|l|}{ Three objects } \\
\hline Same object/adjacent & 760 & 159 & 3.9 & 1,129 & 258 & 5.5 \\
\hline Same object/separated & 829 & 156 & 4.3 & 1,177 & 277 & 1.7 \\
\hline Different objects/different types & s 825 & 179 & 2.3 & 1,143 & 211 & 0.6 \\
\hline Different objects/same type & 926 & 174 & 4.2 & 1,295 & 270 & 3.4 \\
\hline \multicolumn{7}{|l|}{ Abstract Matching 1} \\
\hline Level 1 & 749 & 158 & 1.7 & 1,408 & 381 & 1.0 \\
\hline Level 2 & 1,149 & 368 & 3.3 & 2,321 & 748 & 4.2 \\
\hline Level 3 & 1,316 & 360 & 2.6 & 2,656 & 794 & 3.0 \\
\hline \multicolumn{7}{|l|}{ Abstract Matching 2} \\
\hline Level 1 & 800 & 182 & 1.9 & 1,282 & 229 & 0.7 \\
\hline Level 2 & 1,238 & 328 & 6.0 & 2,338 & 570 & 5.7 \\
\hline Level 3 & 1,481 & 410 & 6.3 & 2,921 & 804 & 6.2 \\
\hline
\end{tabular}

one of the top two objects was identical to the sample on one of the dimensions (the relevant dimension), whereas the other object differed from the sample on this dimension. The remaining (irrelevant) dimensions were either held constant across or varied across all three objects, depending on the condition. Level of difficulty increased with the number of irrelevant, varied dimensions.

Abstract Matching Task 2. The stimulus consisted of three arrays of objects; each array appeared within a $6.2^{\circ}$ square. Participants were instructed to indicate which of the upper two arrays was the best match to the lower array (Figure 1, bottom panel) by pressing the corresponding button. Arrays varied on four dimensions: number $(2,3$, or 4$)$, shape (circle, triangle, or square), color (red, blue, or yellow), and orientation (vertical, horizontal, or diagonal). On any given trial, one of the top two arrays (the match) was identical to the sample on one dimension (the relevant dimension), whereas the other array (the foil) did not match the sample on this dimension. The remaining (irrelevant) dimensions were either held constant across all three arrays or were varied across all three arrays, depending on the condition.

\section{Procedure}

Each participant was given general instructions at the beginning of the session and both specific instructions and practice trials prior to each task. Participants were instructed to respond as quickly and accurately as possible on all tasks. The first two experimental trials were buffers and were not included in any analyses. All tasks had 24 trials in each experimental condition (see the left column of Table 1). All trials began with presentation of a fixation point. When the participant pressed the bottom button on the response panel, the stimulus for the current trial was presented after a 200msec delay and remained on the screen until a response occurred. The screen then remained blank for $700 \mathrm{msec}$. The computer beeped if a participant made an error or failed to respond within $10 \mathrm{sec}$.

\section{RESULTS}

Accuracy was generally high (better than 93\%) for both groups, and age differences in accuracy were small (Table 1). Averaged across conditions, the age difference was less than $2 \%$ on all tasks. Only correct RTs were included in the analyses. The correlation between the mean RTs for young adults and older adults in corresponding conditions was .980 , indicating that experimental manipulations had extremely similar effects on task difficulty for young and older adults. The older adult group was slower than the younger adult group in all conditions of all tasks, and the size of the age difference in RTs increased systematically with task difficulty from less than $200 \mathrm{msec}$ on the two easiest (one-arrow and one-object) tasks to more than 1,000 msec on the two most complex conditions of the two most difficult (abstract matching) tasks. The equation for the regression of the older adult group's mean RTs on those of the young adult group was $\mathrm{RT}_{\mathrm{o}}=2.27 * \mathrm{RT}_{\mathrm{y}}-0.483 \mathrm{~s}$, indicating that across the seven visuospatial tasks, the older adults' cognitive processing was approximately twice as slow, on the average, as that of the young adults.

Analogous complexity effects were observed in the performance of individual participants. Figure 2 shows the data from the fastest and slowest individuals from each age group. Like the age difference in RTs, the size of the difference between an individual's RTs and the average RT for his/her age increased systematically with task dif- 

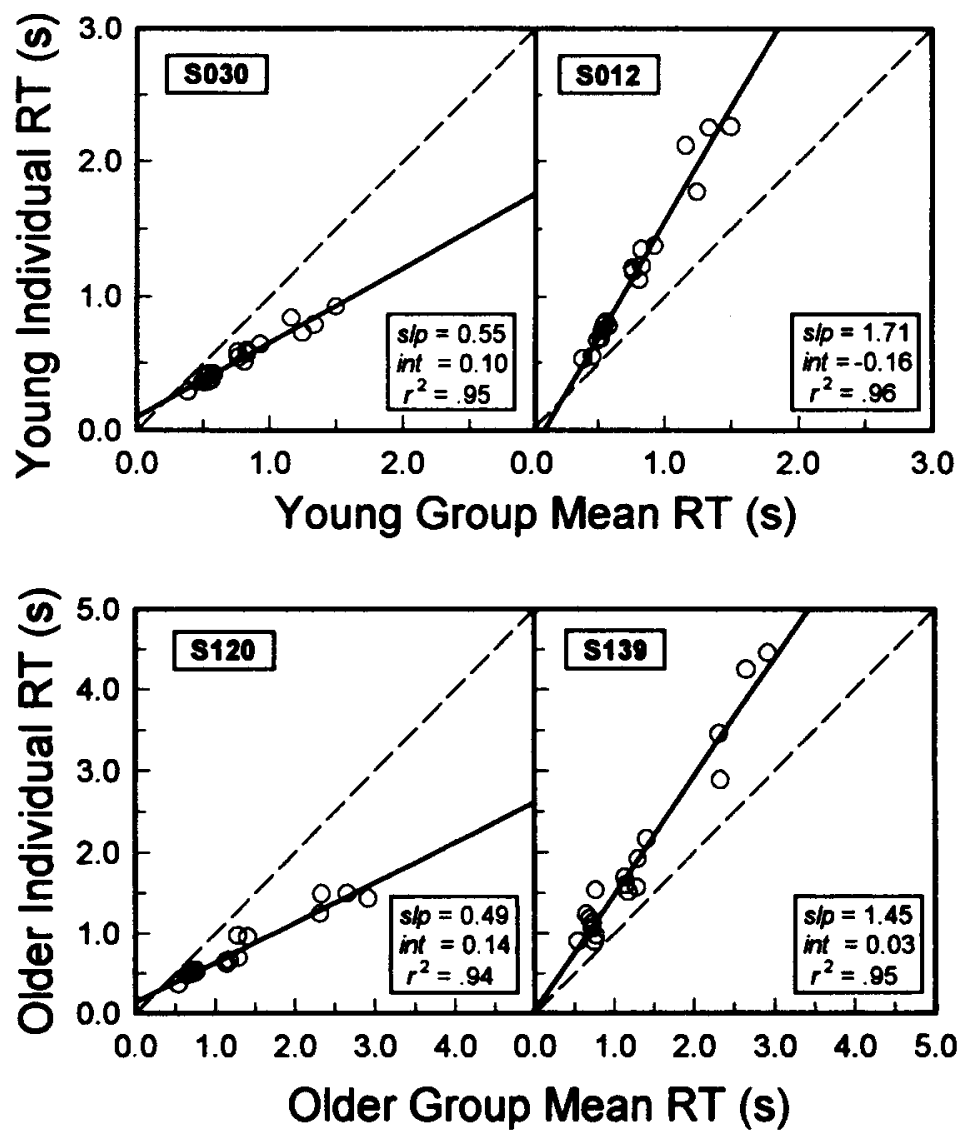

Figure 2. Individual adults' mean response times (RTs) as a function of the mean RT for their age group. The RTs and best-fitting linear functions for the fastest and slowest participants from each age group are shown. The solid lines represent the linear functions that best fit the individual data. The regression parameters $($ slope $=s l p$; intercept $=i n t)$ and proportion of explained variance $\left(r^{2}\right)$ are given for each participant. If an individual's RTs did not differ from the average, the data would fall along the dashed line.

ficulty. As predicted by Equation 2, the relations between the RTs of these 4 subjects and those of their peers were well described by linear functions. Strong linear relations between individuals' RTs and those of their peers were also observed for most other participants: For younger individuals, the median $r^{2}$ was .96 (semi-interquartile range $=0.015$ ); for older individuals, the median $r^{2}$ for was .98 (semi-interquartile range $=0.014$ ).

The finding that individuals' RTs are simple linear functions of the averages for their age group indicates that individuals tend to be consistently fast or consistently slow across diverse tasks. Results obtained from factor analyses of the RTs for the seven tasks provided further evidence of this consistency. RTs on all seven tasks were highly correlated (Table 2), and a single significant principal component (eigenvalue $=5.88$ ) loaded heavily on all tasks and accounted for $84 \%$ of the variance in standard scores.

Similar results were obtained when the RTs for younger and older adults were analyzed separately. To determine whether the composition of the general speed factor was age invariant, we calculated the correlation between the observed factor scores of the individual older adults and the factor scores that would be predicted for the older adults based on the factor loadings determined from the young adults' data. The correlation between the observed and predicted scores of older adults was .99 , suggesting that the composition of the general speed factor was equivalent for both age groups (Gorsuch, 1984).

To test the prediction that age-related slowing increases the absolute size of differences within an age group simply by magnifying individual differences in processing efficiency, each group was divided into fast, average, and slow subgroups as follows: For each age group, $z$ scores were calculated for each experimental condition, and the mean $z$ score was determined for each participant in that group. A participant's mean $z$ score then served as an index of his/her overall performance, and participants were ranked on their mean $z$ scores. The top and bottom 13 participants formed the slow and fast subgroups, respectively; the middle 14 participants formed the medium subgroup. 
Table 2

\begin{tabular}{|c|c|c|c|c|c|c|c|}
\hline Task & Loadings & 1 & 2 & 3 & 4 & 5 & 6 \\
\hline 1. Arrow direction & .919 & & & & & & \\
\hline 2. One object & .911 & .891 & & & & & \\
\hline 3. Disjunctive choice & .935 & .876 & .825 & & & & \\
\hline 4. Two objects & .961 & .886 & .905 & .903 & & & \\
\hline 5. Three objects & .928 & .832 & .793 & .874 & .876 & & \\
\hline 6. Abstract Matching 1 & .855 & .658 & .672 & .720 & .766 & .774 & \\
\hline 7. Abstract Matching 2 & .905 & .742 & .748 & .793 & .818 & .803 & .916 \\
\hline
\end{tabular}

When the mean RTs of the fast and slow subgroups were regressed on their corresponding group mean RTs (Figure 3), there were no significant differences between the regression parameters for the fast young and fast older subgroups or between the regression parameters for the slow young and slow older subgroups (all $t \mathrm{~s}<1$ ). All data points were accurately described by two functions, one for the fast subgroups (slope $=0.74$ ) and one for the slow subgroups (slope $=1.23$ ); both $r^{2}>.99$. These results reveal that not only were both the fast subgroups more efficient than average for their age on all tasks, but also (and as predicted by the derivation of Equation 2), both young and older fast subgroups were more efficient to approximately the same degree. Similarly, both slow subgroups were less efficient than average for their age on all tasks, and (again, as predicted) both young and older slow subgroups were less efficient to approximately the same degree.

Finally, the RTs of the older fast subgroup were regressed on the RTs of the younger fast subgroup, the RTs of the older medium subgroup were regressed on the RTs of the younger medium subgroup, and the RTs of the older slow subgroup were regressed on the RTs of the younger slow subgroup. Tests for separate regressions revealed no significant differences among the parameters for the fast, medium, and slow subgroups (all $t \mathrm{~s}<1$ ), and a single linear function $\left(\mathrm{RT}_{\mathrm{o}}=2.14 * \mathrm{RT}_{\mathrm{y}}-0.390 \mathrm{~s}\right)$ provided a very good description $\left(r^{2}=.94\right)$ of the relation between the RTs of older and younger adults of corresponding ability. The slope of the linear regression equation suggests that the cognitive processing of each subgroup of older adults was slowed by factor of approximately 2 relative to the cognitive processing of the corresponding subgroup of younger adults.

\section{DISCUSSION}

For both young and older adults, orderly relationships were observed between the RTs of individual fast and slow information processors and the mean RTs for their age group such that individual performance could be predicted from average performance without regard to the nature of the task. The present results replicate Hale and Jansen's (1994) findings with respect to young adults using a different set of tasks. More importantly, they extend these findings to individual differences in processing speed among older adults and suggest that in older adults, as in young adults, the absolute magnitude of individual differences in RTs on different tasks is primarily determined by the amount (rather than the type) of processing, at least within the visuospatial domain.

Factor analyses provided converging evidence for the task independence of individual differences in visuospatial processing speed. For both age groups, there was a general speed factor that loaded nearly equally on all tasks and accounted for most of the variance in standardized scores. Moreover, the factor scores of the older adults, derived from analysis of data from their group only, could be predicted precisely based on the task loadings of the single speed factor obtained from analysis of the young adults' data. These results are consistent with previous reports that when young adults are tested on a battery of speeded tasks, principal component analyses show only a single speed factor (e.g., Hale \& Jansen, 1994; Miller \& Vernon, 1992; Vernon \& Jensen, 1984). The present results extend this finding to older adults and suggest further that the structure of the general visuospatial speed factor is age invariant.

The presence of a single speed factor that loads equivalently on all tasks indicates that individuals maintain their ranks with respect to speed from task to task. It says little, however, about the absolute size of the differences in performance on different tasks, which is precisely what the present investigation tells us about. Importantly, this study provides a straightforward test of the magnification hypothesis and its predictions regarding the effect of aging on the size of individual differences. If the magnification hypothesis is correct, individual RTs should be a linear function of group mean RTs for the same tasks and conditions, and the parameters of the relation between individual (or subgroup) performance and average performance for an age group should be approximately equal for matched young and older individuals or subgroups. This is precisely what was observed in the present study: Tests for separate regressions revealed no significant age differences in the relationship of the RTs of subgroups of fast and slow processors to the average RTs for their peers.

Regression of the mean RTs of each older subgroup on the mean RTs of the corresponding young subgroup provides further information regarding the effect of aging on the processing efficiency of older adults. As indexed by the regression slopes, the visuospatial processing of each 


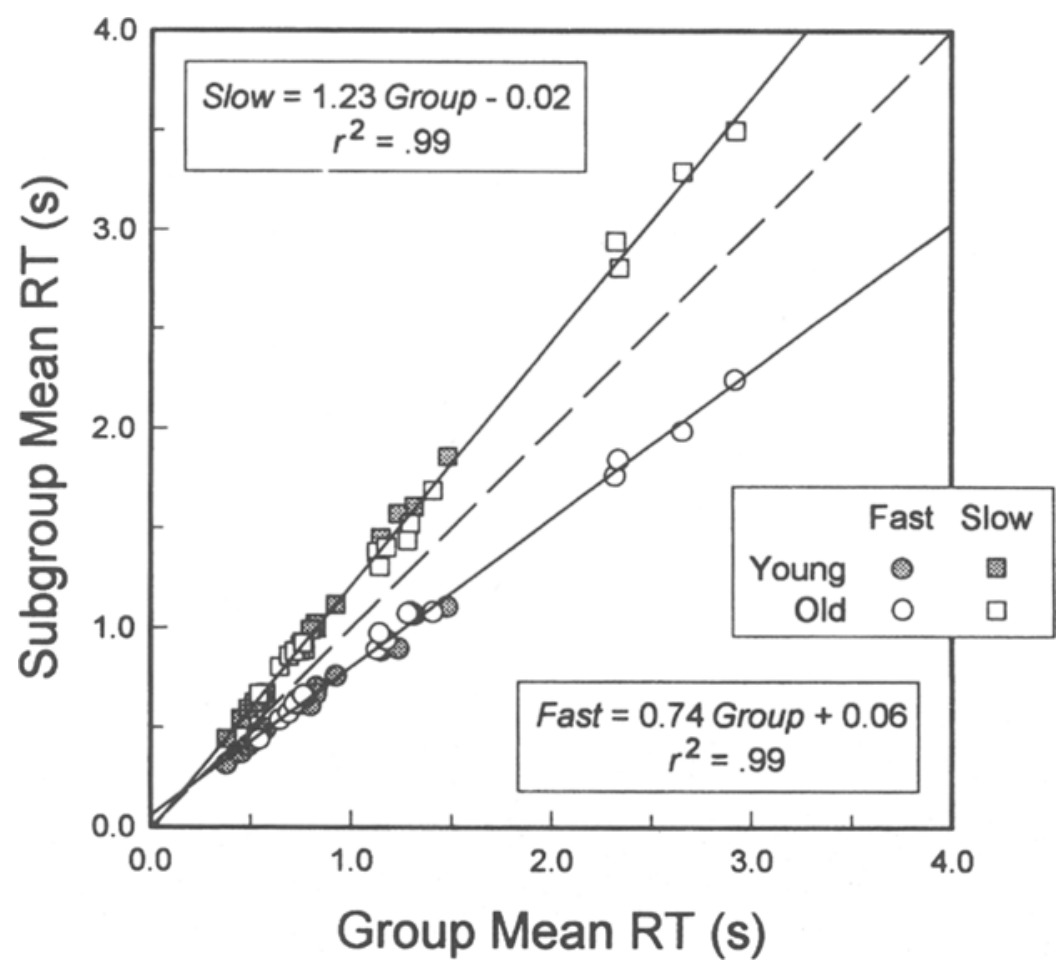

Figure 3. Mean response times (RTs) of the fast younger, fast older, slow younger, and slow older subgroups as a function of their corresponding age groups' mean RT. One solid line represents the linear function that best fit the data for both the older and younger fast subgroups; the other solid line represents the linear function that best fit the data for both the older and younger slow subgroups. If there were no differences among the RTs for the fast and slow younger and older adult subgroups and the mean RTs for their age groups, the data would fall along the dashed line.

of the three subgroups (slow, medium, and fast) of older adults was slowed by a factor of approximately 2 relative to the corresponding subgroup of young adults. This finding is consistent with previous estimates of age-related slowing provided by meta-analyses (Lima, Hale, \& Myerson, 1991; Sliwinski \& Hall, 1998) of age differences on a variety of visuospatial tasks (e.g., visual search, mental rotation). This twofold slowing of visuospatial processing in older adults makes the present finding of equivalent relations between individual and average RTs in young and older adults all the more remarkable, and the fact that the magnification hypothesis predicts such relations would appear to provide strong evidence for its validity.

Are the present findings regarding individual differences peculiar to the specific tasks used in this study? Such findings might be expected on highly similar tasks differing only in complexity (e.g., visual search tasks with different display sizes). The present tasks, however, were selected to tap a diverse set of perceptual and cognitive processes. Some tasks presented only two stimuli (e.g., a left or a right arrow), and participants simply discriminated between them. Other tasks involved making judgments about relationships (e.g., whether two arrows pointed in the same direction). Yet other tasks involved more abstract relationships (e.g., how many objects were the same shape or which of two multidimensional stimuli matched a third stimulus on more dimensions), and the stimuli were never repeated. Since the present study was completed (Myerson, Zheng, \& Hale, 1996), we have reanalyzed data from even more diverse tasks, both visuospatial and verbal (Hale, Myerson, Faust, \& Fristoe, 1995; Myerson, Hale, Chen, \&, Lawrence, 1997), and it appears that the present findings are robust and generalize to a wide variety of information processing tasks.

The present findings may have implications that extend well beyond age and individual differences in RTs. Salthouse (1996) has argued that speed is a fundamental mediator of age differences in a variety of cognitive abilities. If so, the fact that age magnifies individual differences in speed suggests that aging may also amplify individual differences in other, nonspeeded, aspects of cognitive ability (e.g., memory and reasoning). Thus, individuals of lower ability, who tend to be slower processors (see, e.g., Miller \& Vernon, 1992), should show greater cognitive decline with age than faster processors of higher ability. Consistent with this view, Shimamura, Berry, Mangels, Rusting, and Jurica (1995) reported that older college professors showed smaller age-related cognitive deficits than older adults who were not professors. As Shimamura et al. noted, however, it is possible that the 
high level of mental activity engaged in by professors acts to minimize age-related declines or that this mental activity leads to developing strategies that compensate for the effects of biological aging. Further studies specifically designed to distinguish between these interpretations are needed.

In addition, the present findings may have implications for the interaction of other individual difference variables (e.g., psychological or physical health status). For example, recent studies of cognitive change in depression (White, Myerson, \& Hale, 1997) and traumatic brain injury (Ferraro, 1996) have reported general slowing similar to that observed in older adults. That is, regression of the RTs of depressed or brain-injured groups on those of controls reveals linear relations suggestive of task-independent decreases in processing efficiency. Similar findings have also been reported for multiple sclerosis (Kail, 1997) and dementia of the Alzheimer type (Myerson, Lawrence, Hale, Jenkins, \& Chen, 1998).

If the present findings generalize to such cases (e.g., if declines in health status also magnify individual differences), then individuals who were slow when they were healthy may be more affected by a variety of insults, such as depression and brain injury. Consistent with this view, Maylor and Rabbitt (1993) have reported results suggesting that the effect of alcohol on RTs is greater for slower individuals than for faster individuals. Similarly, older adults may be more affected by biological insults, not just because they are older and perhaps lacking in recuperative powers or reserve capacity, but simply because they are slower.

The current theoretical effort builds on previous work by Cerella $(1985,1990)$ and Hale and Jansen (1994). Although future theoretical developments will no doubt require more complicated models (e.g., Myerson \& Adams, 1998; Ratcliff, Spieler, \& McKoon, 2000), the mathematical representation of age and individual differences presented here is deliberately simplified. A simplified treatment was selected for this initial effort in order to obtain clear, testable predictions whose limitations might be corrected in subsequent efforts. Few such limitations were apparent, but it is quite possible that they might be revealed by more fine grained analyses in future studies. Nevertheless, the present finding that most of the variance could be explained by simple, theoretically derived linear regression equations is consistent with the magnification hypothesis and provides support for the present theoretical framework.

\section{REFERENCES}

Cerella, J. (1985). Information processing rates in the elderly. Psychological Bulletin, 98, 67-83.

Cerella, J. (1990). Aging and information-processing rate. In J. E. Birren \& K. W. Schaie (Eds.), Handbook of the psychology of aging (3rd ed., pp. 201-221). San Diego: Academic Press.

Cerella, J., \& Hale, S. (1994). The rise and fall of information processing rates over the life span. Acta Psychologica, 86, 109-197.

Cerella, J., Poon, L. W., \& Williams, D. M. (1980). Age and the complexity hypothesis. In L. W. Poon (Ed.), Aging in the 1980s: Psycho- logical issues (pp. 332-340). Washington, DC: American Psychological Association.

Ferraro, F. R. (1996). Cognitive slowing in closed-head injury. Brain \& Cognition, 32, 429-440.

Gorsuch, R. L. (1984). Factor analysis. Philadelphia: W. B. Saunders.

HALE, S., \& JANSEN, J. (1994). Global processing-time coefficients characterize individual differences in cognitive speed. Psychological Science, 5, 384-389.

hale, S., Myerson, J., Faust, M., \& Fristoe, N. (1995). Converging evidence for domain-specific slowing from multiple nonlexical tasks and multiple analytic methods. Journal of Gerontology: Psychological Sciences, 50, P202-P211.

Hale, S., Myerson, J., Smith, G. A., \& Poon, L. W. (1988). Age, variability, and speed: Between-subjects diversity. Psychology \& Aging, 3, 407-410.

HerTzog, C., \& Schaie, K. W. (1986). Stability and change in adult intelligence: 1 . Analysis of longitudinal covariance structures. Psychology \& Aging, 1, 159-171.

KAIL, R. (1997). The neural noise hypothesis: Evidence from processing speed in adults with multiple sclerosis. Aging, Neuropsychology, \& Cognition, 4, 157-165.

KaIL, R., \& Salthouse, T. A. (1994). Processing speed as a mental capacity. Acta Psychologica, 86, 199-225.

Lima, S. D., HAlE, S., \& Myerson, J. (1991). How general is general slowing? Evidence from the lexical domain. Psychology \& Aging, 6 , 416-425.

MAYLOR, E. A., \& RABbitT, P. M. A. (1993). Alcohol, reaction time and memory: A meta-analysis. British Journal of Psychology, 84, 301317.

MaYlor, E. A., \& RabBitT, P. M. A. (1994). Applying Brinley plots to individuals: Effects of aging on performance distributions in two speeded tasks. Psychology \& Aging, 9, 224-230.

MiLler, L. T., \& VERNON, P. A. (1992). The general factor in short-term memory, intelligence, and reaction time. Intelligence, 16, 5-29.

Myerson, J., \& Adams, D. R. (1998). Mathematical and connectionist models of individual differences in processing speed. Abstracts of the Psychonomic Society, 3, 49.

Myerson, J., Hale, S., Chen, J., \& Lawrence, B. (1997). General lexical slowing and the semantic priming effect: The roles of age and ability. Acta Psychologica, 96, 83-101.

Myerson, J., Lawrence, B. M., Hale, S., Jenkins, L., \& Chen, J. (1998). General slowing of lexical and nonlexical information processing in dementia of the Alzheimer type. Aging, Neuropsychology, \& Cognition, 5, 1-12.

Myerson, J., ZHENG, Y., \& HALE, S. (1996). Multiplicative interaction of age and ability in individual speeded performance. Abstracts of the Psychonomic Society, 1, 50.

RatClifF, R., SPIELER, D., \& MCKoon, G. (2000). Explicitly modeling the effects of aging on response time. Psychonomic Bulletin \& Review, 7, 1-25.

Salthouse, T. A. (1996). The processing-speed theory of adult age differences in cognition. Psychological Review, 103, 403-428.

Schaie, K. W. (1989). Perceptual speed in adulthood: Cross-sectional and longitudinal studies. Psychology \& Aging, 4, 443-453.

Shimamura, A. P., Berry, J. M., Mangels, J. A., Rusting, C. L., \& JuRICA, P. J. (1995). Memory and cognitive abilities in university professors. Psychological Science, 6, 271-277.

SLIWINSKI, M. J., \& HALL, C. B. (1998). Task-dependent cognitive slowing in the elderly: A meta-analysis using hierarchical linear models with random coefficients. Psychology \& Aging, 13, 164-175.

VERNON, P. A., \& JENSEN, A. R. (1984). Individual and group differences in intelligence and speed of information processing. Personality \& Individual Differences, 5, 411-423.

White, D. A., Myerson, J., \& HaLE, S. (1997). How cognitive is psychomotor slowing in depression? Evidence from a meta-analysis. Aging, Neuropsychology, \& Cognition, 4, 166-174.

(Manuscript received March 25, 1998; revision accepted for publication April 20, 1999.) 